\title{
Angle gathers in wave-equation imaging for transversely isotropic media ${ }^{a}$
}

${ }^{a}$ Published in Geophysical Prospecting, 59, 422-431, (2011)

Tariq Alkhalifah, King Abdullah University for Science and Technology, Sergey Fomel, The University of Texas at Austin

\begin{abstract}
In recent years, wave-equation imaged data are often presented in common-image angle-domain gathers as a decomposition in scattering angle at the reflector, which provide a natural access to analyzing migration velocities and amplitudes. In the case of anisotropic media, the importance of angle gathers is enhanced by the need to properly estimate multiple anisotropic parameters for a proper representation of the medium. We extract angle gathers for each downwardcontinuation step from converting offset-frequency planes into angle-frequency planes simultaneously with applying the imaging condition in a transversely isotropic with a vertical symmetry axis (VTI) medium. The analytic equations, though cumbersome, are exact within the framework of the acoustic approximation. They are also easily programmable and show that angle gather mapping in the case of anisotropic media differs from its isotropic counterpart, with the difference depending mainly on the strength of anisotropy. Synthetic examples demonstrate the importance of including anisotropy in the angle gather generation as mapping of the energy is negatively altered otherwise. In the case of a titled axis of symmetry (TTI), the same VTI formulation is applicable but requires a rotation of the wavenumbers.
\end{abstract}

\section{INTRODUCTION}

Angle gathers have gained prominence as they provide wave equation imaging methods with an outlet to perform velocity analysis. Angle gathers also alleviate the limitations that offset gathers have in handling multi pathing (de Bruin et al., 1990; Mosher and Foster, 2000; Stolk and de Hoop, 2006). An angle gather decomposition for anisotropic media will allow us to export these features to the anisotropic world, and this is especially important considering the number of parameters we need to deal with in anisotropic media and the prevalent multi-pathing that takes place in such media.

Downward wave extrapolation provides an accurate method of seismic imaging in structurally complex areas. Downward wave extrapolation is also naturally formulated to produce angle gathers (de Bruin et al., 1990; Mosher and Foster, 2000; Rickett 
and Sava, 2002; Xie and Wu, 2002; Sava and Fomel, 2003; Soubaras, 2003; Biondi and Symes, 2004; Sava and Fomel, 2005). Fomel (2004) showed that structural dependence can be removed in a depth-slice approach to extracting angle gathers. Specifically, one can generate gathers at each depth level, converting offset-space-frequency planes into angle-space planes and applying simultaneously the imaging condition. The improved mapping retains velocity dependence but removes the effect of the structure. Because of its ray-parameter-based (Fourier) formulation, this approach lends itself naturally to an anisotropic phase-velocity extension.

Migration velocity analysis in anisotropic media remains a challenging and open issue. Multiple parameters are needed to represent the anisotropic model. For a transversely isotropic medium with vertical axis of symmetry (VTI media), only NMO velocity $(v)$ and the non-elliptic parameter $(\eta)$ predominantly influence imaging (Alkhalifah and Tsvankin, 1995; Alkhalifah et al., 2001). Vertical velocity $\left(v_{z}\right)$ controls mainly placement of the image in depth. Nevertheless, estimating even two parameters that can vary laterally and vertically from image gathers is difficult. Angle gathers provide an opportunity to use residual moveout to help update anisotropic parameters. Biondi (2007) suggested an approach to extracting angle gathers in anisotropic media from post-migration data. Biondi's formulation is based on numerical calculation of angle gathers and relies on ray information that is hard to examine analytically.

In this paper, we develop an analytical formulation for extracting angle gathers in VTI 2D media. We use the depth-slice approach to angle gathers as a platform for the extension to VTI. Angle gather mapping depends strongly on anisotropic parameters. We analyze this dependency using numerical computations. Next, we show synthetic data examples that confirm the theoretical analysis. Finally, we explain the possible extension of our approach to TTI (tilted transversely isotropic) media. An extension to 3D can be achieved along the lines of Fomel (2004).

\section{THE DEPTH SLICE APPROACH FOR VTI MEDIA}

Relations between image coordinates and reflection (scattering) angles at reflecting interfaces can be extracted by analyzing the geometry of reflections in the simple case of a dipping reflector in a locally homogeneous medium (Fomel, 2004). The geometry of the reflection ray paths in 2-D is depicted in Figure 1(a).

According to elementary rules of geometry for the ray configuration in Figures 1(a) and $1(\mathrm{~b})$, with the wavenumber vector given by $\mathbf{k}=\omega \mathbf{p}$ as it relates to the rayparameter vector for a given angular frequency $\omega$, opening (scattering) phase angle $\theta$ is represented by the following relation (Fomel, 2004; Sava and Fomel, 2005)

$$
k_{\mathrm{hx}}^{2}+k_{\mathrm{hz}}^{2}=k_{s}^{2}+k_{r}^{2}-2 k_{r} k_{s} \cos (\theta)
$$

where $k_{\mathrm{hx}}$ and $k_{\mathrm{hz}}$ are horizontal and vertical components of the offset wave number, and $k_{s}$ and $k_{r}$ are source and receiver wavenumber amplitudes related to their components as follows: $k_{s}^{2} \equiv k_{\mathrm{sx}}^{2}+k_{\mathrm{sz}}^{2}, k_{r}^{2} \equiv k_{\mathrm{rx}}^{2}+k_{\mathrm{rz}}^{2}$, with $k_{\mathrm{hx}} \equiv k_{\mathrm{rx}}-k_{\mathrm{sx}}, k_{\mathrm{mx}} \equiv k_{\mathrm{rx}}+k_{\mathrm{sx}}$, 


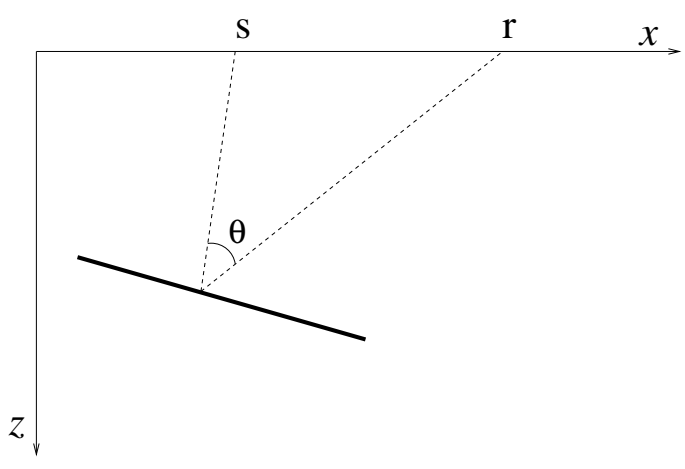

(a)

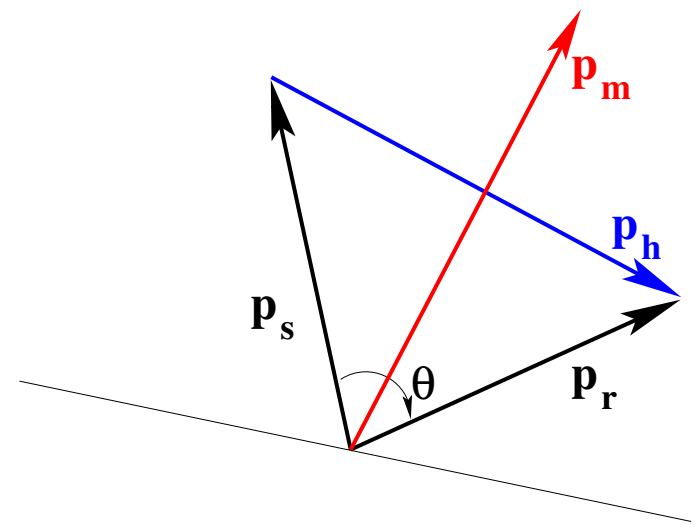

(b)

Figure 1: (a) A schematic plot showing angle $\theta$. Although the model depicts a homogeneous setting, the development will rely on the ray parameters defined in the immediate vicinity of the the reflection point, as shown in b. (b) A schematic plot depicting the relation between the source and receiver ray-parameter vectors $\left(\mathbf{p}_{s}\right.$ and $\left.\mathbf{p}_{g}\right)$ and the offset and midpoint vectors $\left(\mathbf{p}_{h}\right.$ and $\left.\mathbf{p}_{m}\right)$

as suggested by Figure 1(b), where $k_{\mathrm{mx}}$ is the horizontal component of the midpoint wavenumber.

To complete the system of equations necessary to relate angle $\theta$ to midpoint and offset horizontal wavenumbers, we use the dispersion relation developed by Alkhalifah (1998) to define each of $k_{\mathrm{sz}}$ and $k_{\mathrm{rz}}$ as follows:

$$
\begin{aligned}
& k_{\mathrm{sz}}^{2} \equiv\left(\omega \frac{\partial t_{\mathrm{s}}}{\partial z}\right)^{2}=\frac{\omega^{2}}{v_{z}^{2}}-\frac{v^{2} \omega^{2}\left(k_{\mathrm{hx}}-k_{\mathrm{mx}}\right)^{2}}{2 v_{z}^{2}\left(2 \omega^{2}-v^{2} \eta\left(k_{\mathrm{hx}}-k_{\mathrm{mx}}\right)^{2}\right)} \\
& k_{\mathrm{rz}}^{2} \equiv\left(\omega \frac{\partial t_{\mathrm{r}}}{\partial z}\right)^{2}=\frac{\omega^{2}}{v_{z}^{2}}-\frac{v^{2} \omega^{2}\left(k_{\mathrm{hx}}+k_{\mathrm{mx}}\right)^{2}}{2 v_{z}^{2}\left(2 \omega^{2}-v^{2} \eta\left(k_{\mathrm{hx}}+k_{\mathrm{mx}}\right)^{2}\right)},
\end{aligned}
$$

where $v$ is the NMO velocity. Using equation (1) in its expanded form and after some manipulation and collecting terms with the same power of $\cos \theta$, we end up with the following quadratic equation:

$$
a \cos ^{4}(\theta)+b \cos ^{2}(\theta)+c=0
$$

with solutions given by

$$
\theta=\cos ^{-1}\left( \pm \sqrt{\frac{-b \pm \sqrt{b^{2}-4 a c}}{2 a}}\right) .
$$

Analytical representation of the coefficients is shown in Table 1. The four solutions of equation (5) are controlled by the sign of the offset wavenumber and its magnitude compared with the midpoint wavenumber. In the frequency-wavenumber domain, equation (5) can be used to map offset (horizontal) wavenumbers to angle gathers for a specific frequency, midpoint (horizontal) wavenumber, and depth slice. A description 
of an algorithm to use with the mapping equation, in the case of an isotropic medium, is given by Fomel (2004).

Setting $\eta=0$ yields mapping for elliptical anisotropy with coefficients of equation (5) given by Table 2 . The coefficients are represented by much simpler formulas. In the isotropic case, $\eta=0$ and $v_{z}=v$, Table 1 reduces to Table 3 and, if substituted into the mapping formula of equation (5), is equivalent to the corresponding mapping equation of Fomel (2004).

\section{NUMERICAL TESTS: THE ANISOTROPY INFLUENCE}

Using equation (5) we evaluated angle gathers as a function of offset and midpoint wavenumbers for a given frequency. We tested such mapping for various models using different strengths of anisotropy as we varied $\eta, v_{z}$, and the NMO velocity $v$.

Figures 2-3 show contour density plots of angle as a function of offset and midpoint wavenumbers, for a $60-\mathrm{Hz}$ frequency slice. In Figure 2 the medium is isotropic, with a velocity of $2 \mathrm{~km} / \mathrm{s}$. Clearly, for $k_{\mathrm{hx}}=0$, the angle is zero regardless of the midpoint wavenumber, which is expected, because for zero-offset the scattering or opening angle is equal to zero. Also, we observe that angles decrease with dip (or $k_{m x}$ ) for a given offset wavenumber, which is also expected, because for any offset a scattering angle becomes zero in the case of a vertical reflector. The areas given in white in the Figures 2-5 correspond to regions where the $k_{s z}$ or $k_{r z}$ become complex, and thus represent evanescent waves.

Figure 2: Constant-depth constant-frequency $(60 \mathrm{~Hz})$ slice mapped to opening angles for an isotropic medium with velocity equal to $2 \mathrm{~km} / \mathrm{s}$. Zero-offset wavenumber maps to zero (normal incidence) angle. The four blank corners represent evanescent regions. Negative angles correspond to a switch in the source-receiver direction, and thus, the result is symmetric based on the principal of reciprocity

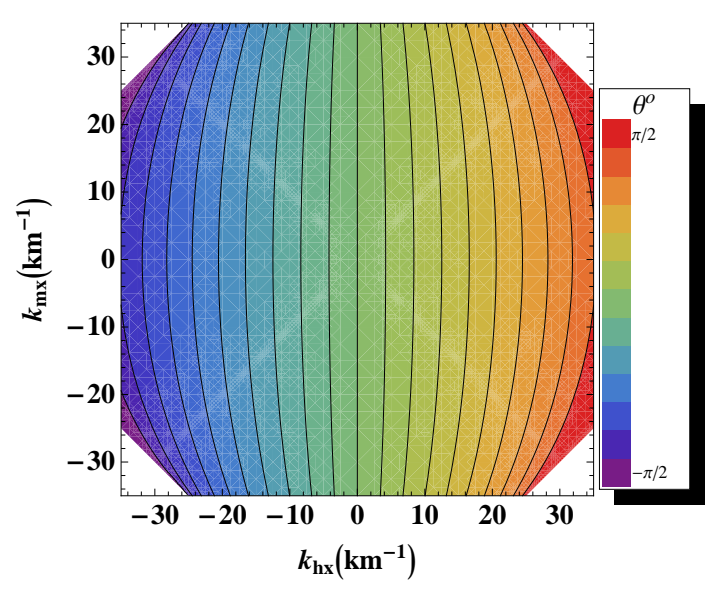

In anisotropic media, as illustrated in Figure 3, for $\eta$ equal to 0.1 and 0.3 , the angles decrease with dip for a constant offset wavenumber faster than in the isotropic case. In the example, considering that $v_{z}$ is lower in the anisotropic models, the 
higher horizontal velocities given by the larger $\eta$ resulted in smaller scattering angles because reflection occurs more updip for larger $\eta$.

Figure 3: Constant-depth constant-frequency $\quad(60 \mathrm{~Hz})$ slice mapped to opening angles as in Figure 2, but for a VTI model with $v_{z}=1.8 \mathrm{~km} / \mathrm{s}, \quad v=2$ $\mathrm{km} / \mathrm{s}$, and $\eta=0.1$ (left) and $\eta=0.3$ (right).
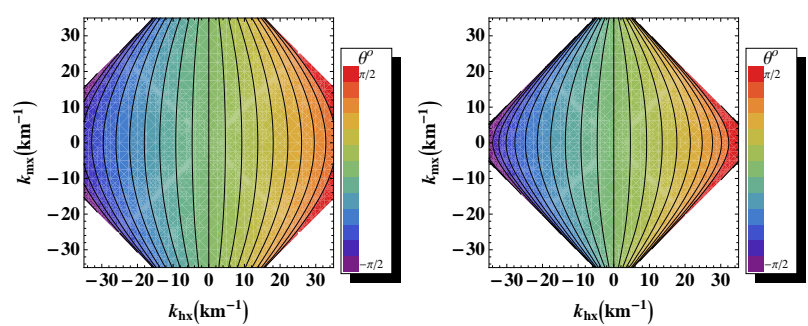

Whereas the influence of $\eta$ is clearly large, the change in vertical velocity has a minor influence on the angles as a function of the midpoint wavenumber (or dip), as demonstrated by the difference plot in Figure 4 . A $0.6 \mathrm{~km} / \mathrm{s}$ difference in vertical velocity of an elliptical isotropic model with $\eta=0$ (left) and a VTI model with $\eta=0.3$ resulted in differences mainly in the offset wavenumber direction, because depth change caused by the different vertical velocity provides variations in angles with offset.

Figure 4: Left: The difference between opening angles for an elliptical anisotropic model with $v_{z}=1.8 \mathrm{~km} / \mathrm{s}, v=2 \mathrm{~km} / \mathrm{s}$ and that of a similar model, with $v_{z}=1.2$ $\mathrm{km} / \mathrm{s}$. Right: The difference between opening angles for a VTI model of Figure 3 (right) for $v_{z}=1.8 \mathrm{~km} / \mathrm{s}, v=2 \mathrm{~km} / \mathrm{s}$, and $\eta=$
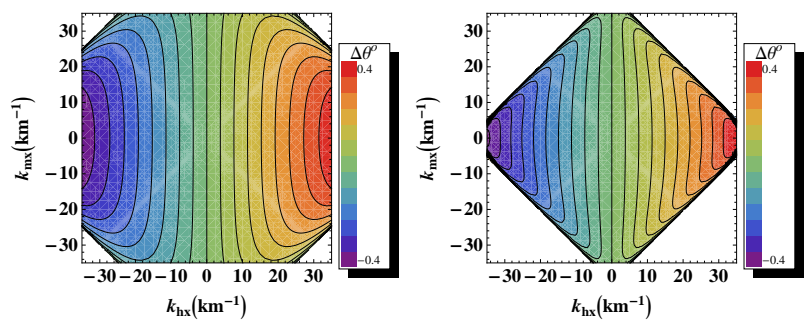
0.3 and that of a similar model, with $v_{z}=1.2 \mathrm{~km} / \mathrm{s}$.

In comparison, if we change the NMO velocity, $v$, the angles hardly change at all, especially around small dips and small offsets. This fact is evident in Figure 5, where we change NMO velocity $0.6 \mathrm{~km} / \mathrm{s}$, and the general difference is small until we get to large offset and midpoint wavenumbers. This difference implies that the mapping is practically NMO-velocity independent. This is the case for $\eta=0$ (left) and $\eta=0.3$ (right) in Figure 5, which implies, that for a given elliptical anisotropic model one can find an isotropic model that produces similar mapping granted that the velocity of the isotropic model is equal to the vertical velocity for the elliptical anisotropy. 

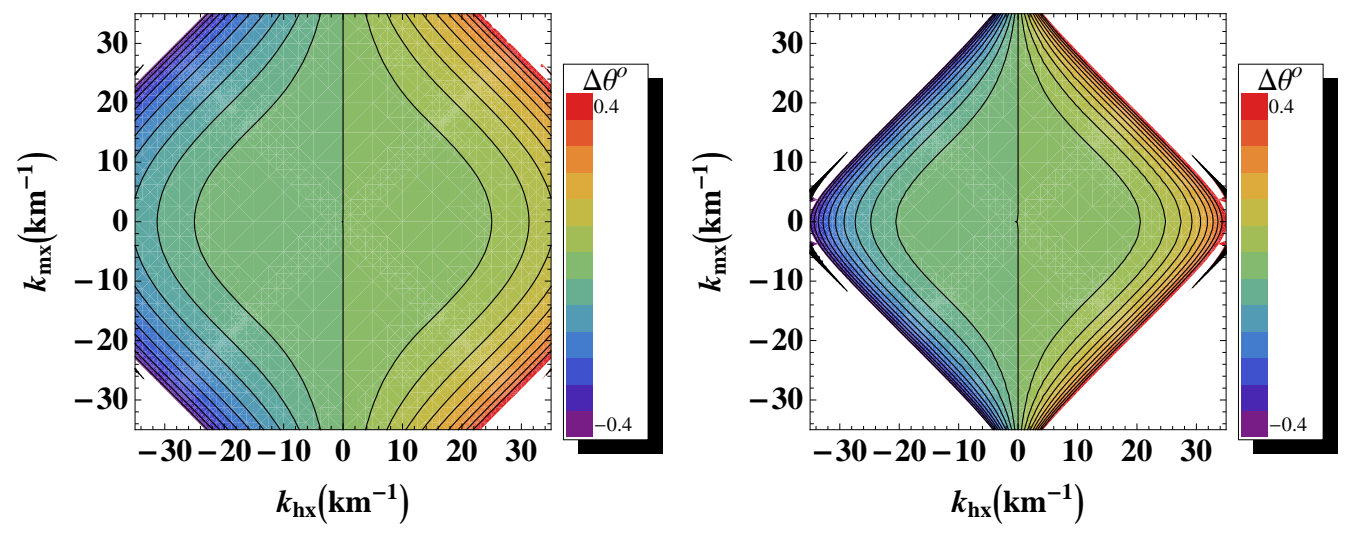

Figure 5: Left: The difference between reflection angles for an elliptical anisotropic model with $v_{z}=1.8 \mathrm{~km} / \mathrm{s}, v=2 \mathrm{~km} / \mathrm{s}$ and that of a similar model, with $v=1.4 \mathrm{~km} / \mathrm{s}$. Right: The difference between reflection angles for a VTI model of Figure 3 (right) for $v_{z}=1.8 \mathrm{~km} / \mathrm{s}, v=2 \mathrm{~km} / \mathrm{s}$, and $\eta=0.3$ and that of a similar model, with $v=1.4$ $\mathrm{km} / \mathrm{s}$.

\section{SYNTHETIC EXAMPLE}

In the following example, we use a homogeneous model for simplicity, although nothing in the development requires that. It is convenient so we can isolate the anisotropy influence on angle gathers decomposition. To follow convention, we display angle gathers in the following examples using half the opening (or scattering) angle $\left(=\frac{\theta}{2}\right)$. We consider the reflector model in Figure 6, which is made up of a number of domelike anticlines. This model allows us to focus on an angle gather located at $8 \mathrm{~km}$ that includes many dips. For a velocity of $2 \mathrm{~km} / \mathrm{s}$ and $\eta=0.2$, we generate the prestack synthetic dataset shown in Figure 7. We use Kirchhoff modeling to obtain the synthetic data (Alkhalifah, 1995). As a reference, we show in Figure 8 the isotropic migration of the isotropic version of the data. In this figure, we observe the extension of reflections that are acquired with a limited offset in the angle representation.

Conventional phase shift downward continuation requires that no lateral velocity variation is present. Since the synthetic model has no lateral (or even vertical) velocity variation, we use a VTI version of the DSR (double-square-root) phase-shift migration (Alkhalifah, 2000) to migrate the data. However, prior to applying the zero-time imaging condition we map the offset wavenumbers to angle, and thus, obtain angle gathers. Figure 9 shows the isotropically migrated section at near zero angle. It also shows on the right hand side the angle gather for an isotropic angle gather mapping. Clearly, the angle gather includes residuals resulting from ignoring anisotropy. These residuals start at the top with deviations at large angles with a fourth-order move- 
out (known as nonhyperbolic) often associated with the semi-horizontal reflectors to second-order strong deviations typically associated with dipping reflectors (Alkhalifah and Tsvankin, 1995). The top plot in Figure 9 is a slice of a constant depth of 2 $\mathrm{km}$ and includes some residual information spanning other angle gathers.

If we downward continue using an anisotropic phase-shift migration followed by an isotropic angle gather mapping, we image the data accurately as shown in Figure 10. Even the angle gathers, despite using an isotropic mapping, show no residuals as we have imaged that data accurately. However, though not immediately obvious, most of the migrated energy is mapped to the wrong angle. On the other hand, an anisotropic mapping of angle gathers places reflections at their true angles (Figure 11). This fact can be realized from comparing the extension of angle gathers of Figures 10 and 11. The lower-than-actual horizontal velocity treatment in the isotropic mapping places energy at smaller reflection angle values. This phenomenon can be directly attributed to the difference between phase and group velocities. Specifically, for horizontal reflections, the isotropic angle gathers map phase angles (ignoring the difference), while anisotropic ones map ray angles. When the horizontal velocity is higher in a VTI medium, the ray angle, measured from vertical, tend to be higher than the phase angle.

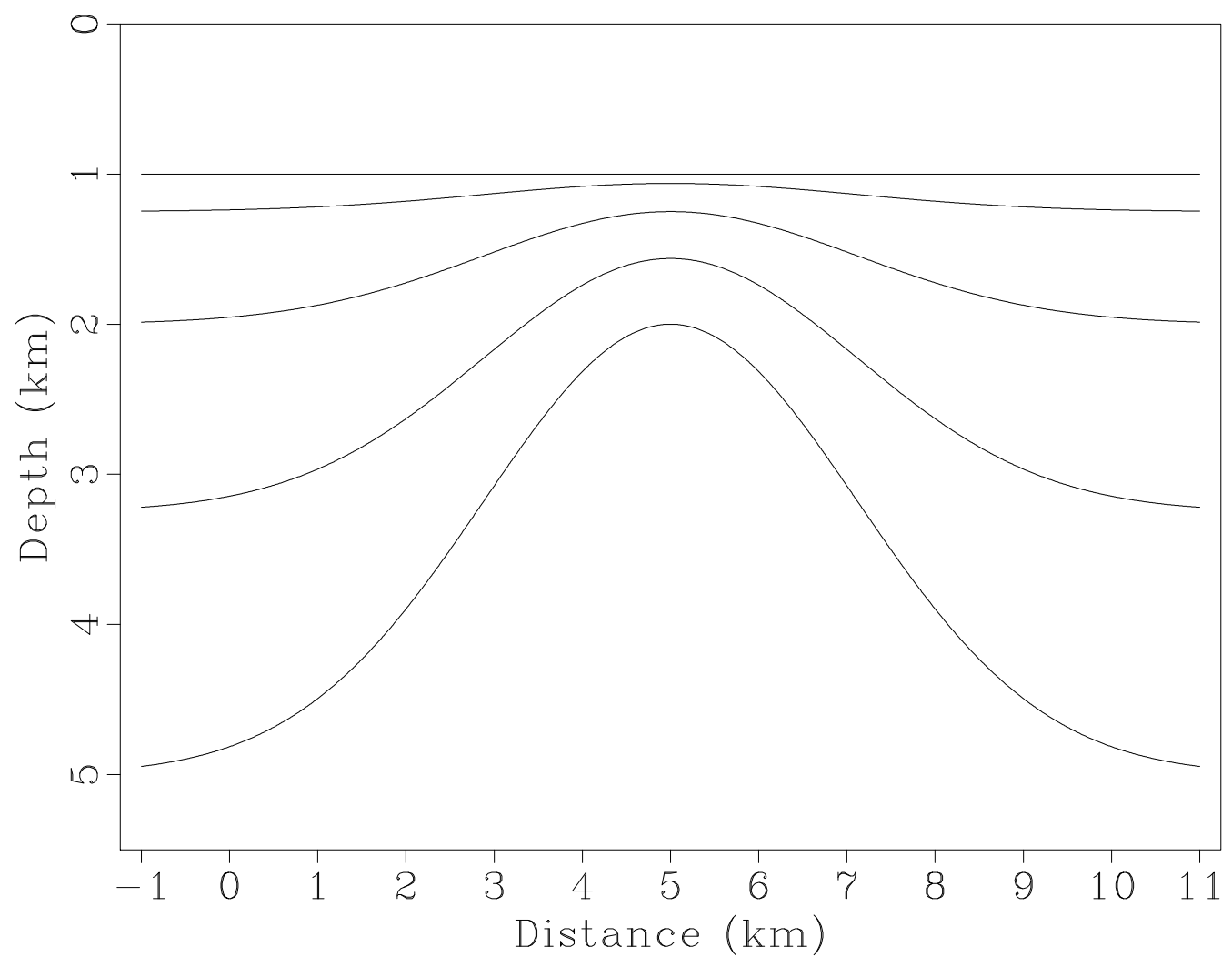

Figure 6: A reflector model containing 5 reflections in a dome like formation. We focus on angle gathers at location $8 \mathrm{~km}$, at which several reflection dips are represented.

This synthetic test the importance of anisotropic angle gather mapping to place 


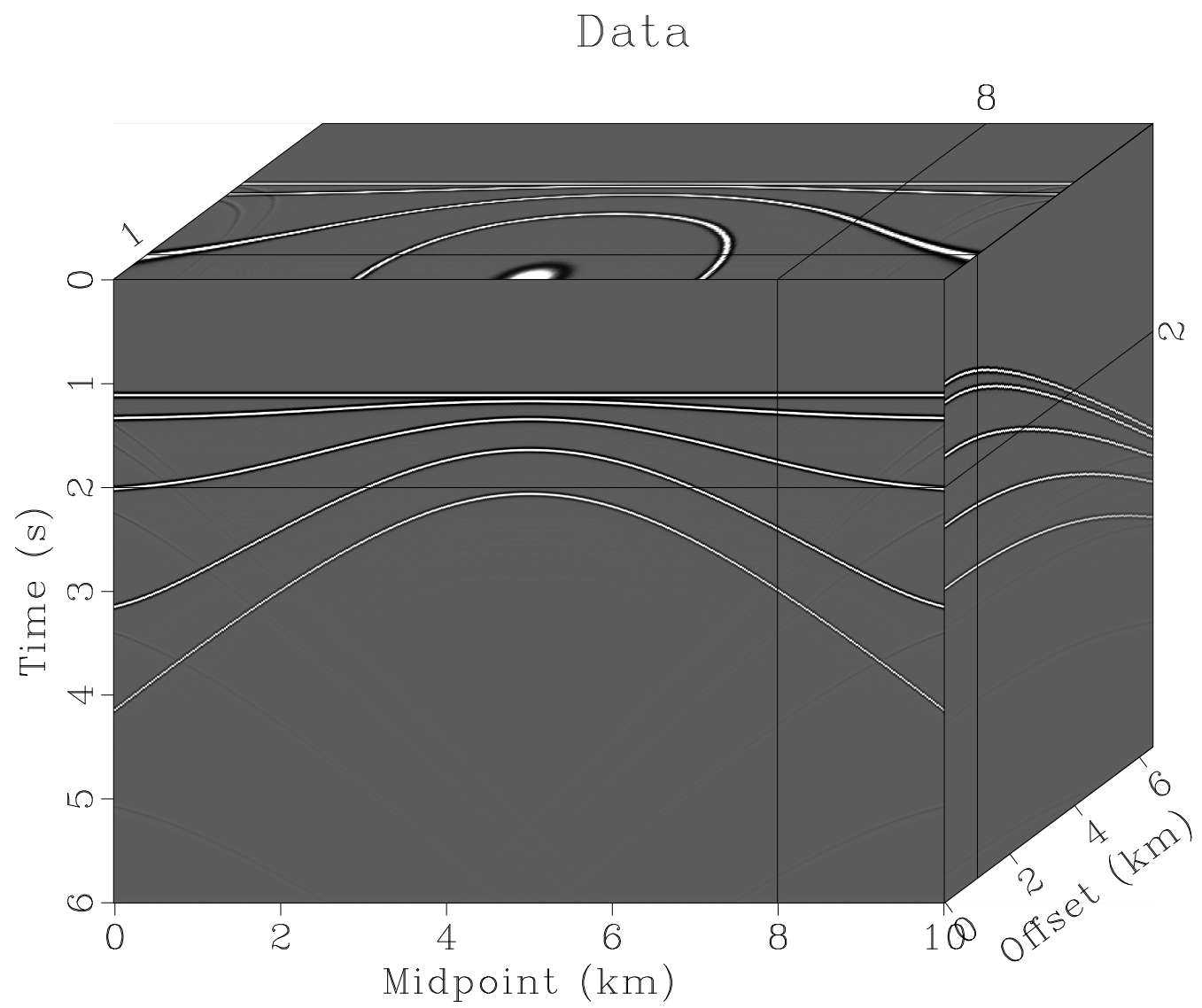

Figure 7: Prestack synthetic data generated using Kirchhoff modeling for a VTI model with velocity (NMO and vertical) equal $2 \mathrm{~km} / \mathrm{s}$ and $\eta=0.2$. 


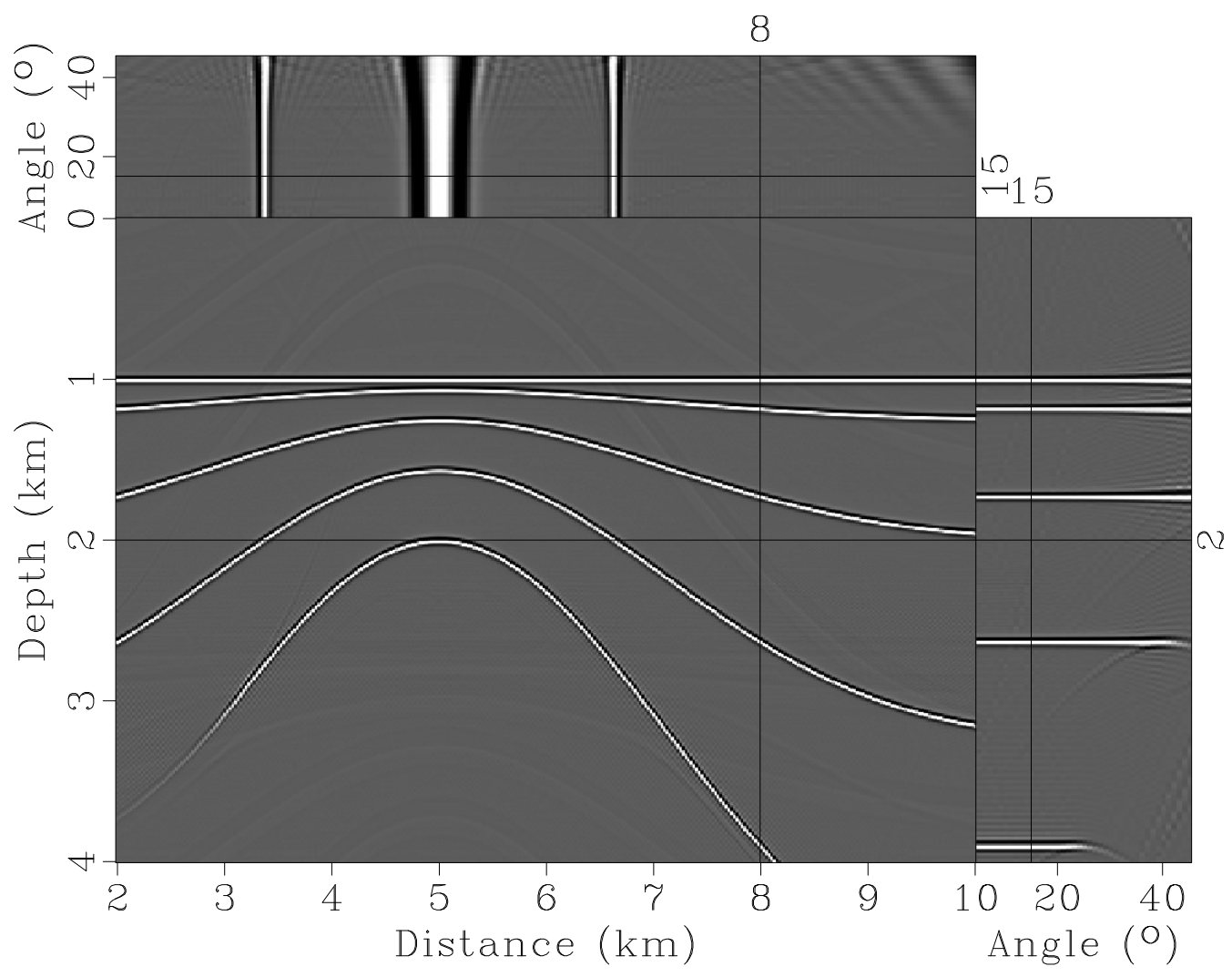

Figure 8: Migrated section after an isotropic migration with velocity of $2 \mathrm{~km} / \mathrm{s}$ of an equivalent isotropic synthetic data. The angle gathers obtained using an isotropic mapping at $8 \mathrm{~km}$ location is displayed on the right. The top section shows a depth slice as a function of angle gather at depth $2 \mathrm{~km}$. 


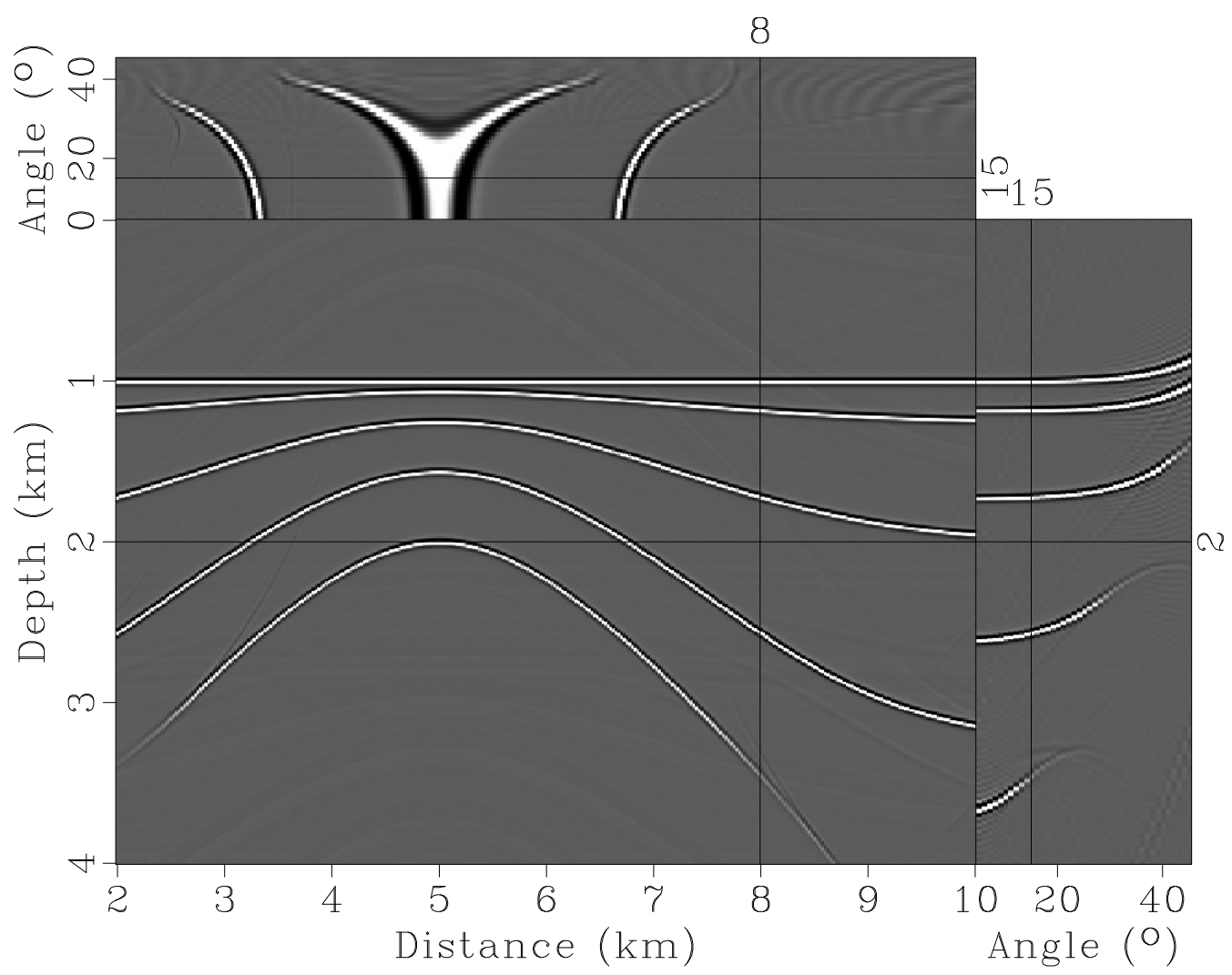

Figure 9: Migrated section after an isotropic migration with velocity of $2 \mathrm{~km} / \mathrm{s}$ of the VTI synthetic data in Figure 7. Again, the angle gathers obtained using an isotropic mapping at $8 \mathrm{~km}$ location is displayed on the right, and the top section shows a depth slice as a function of angle gather at depth $2 \mathrm{~km}$. 


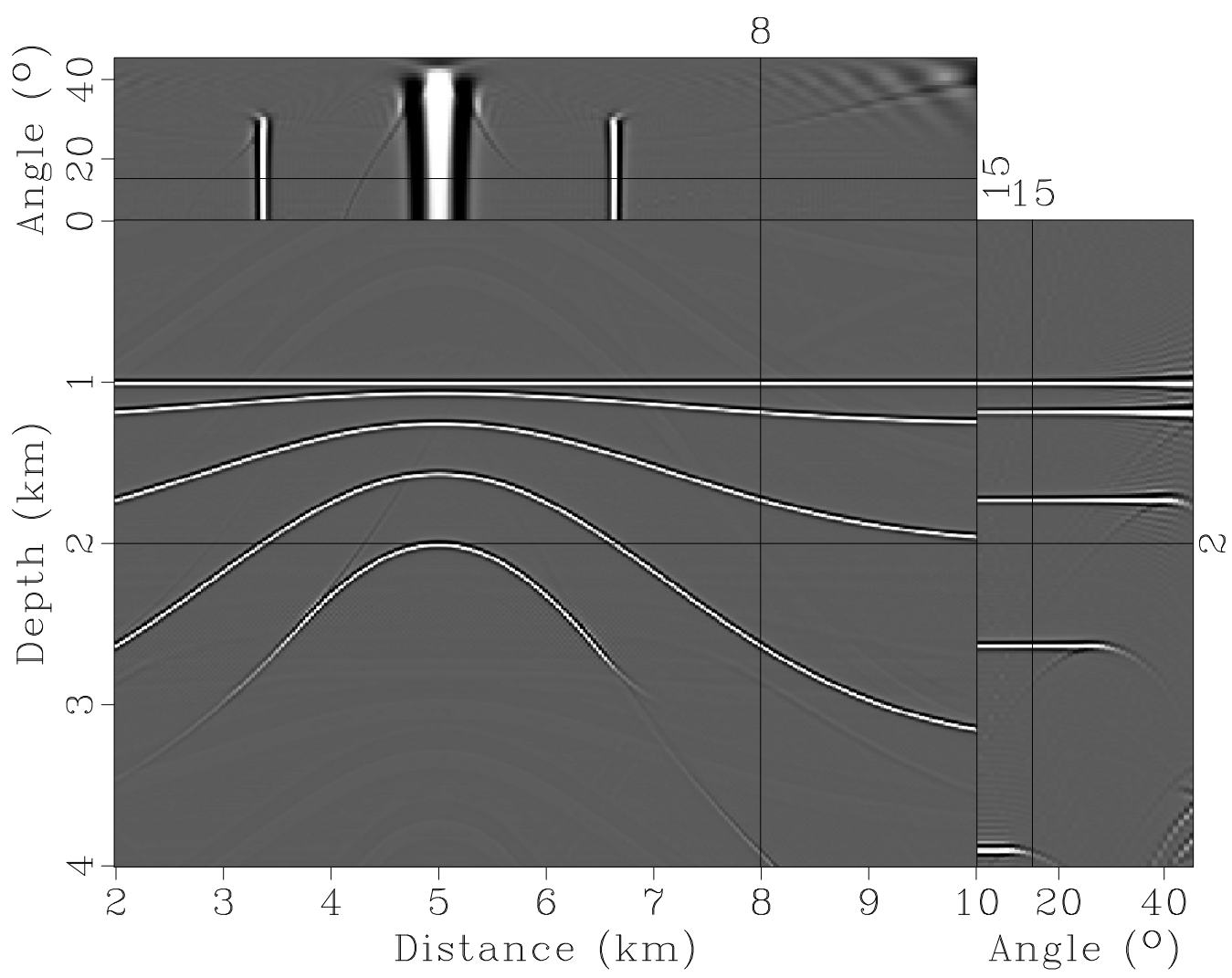

Figure 10: Migrated section after a VTI migration with velocity of $2 \mathrm{~km} / \mathrm{s}$ and $\eta=0.2$ of the VTI synthetic data in Figure 7. Again, the angle gathers obtained using an isotropic mapping at $8 \mathrm{~km}$ location is displayed on the right, and the top section shows a depth slice as a function of angle gather at depth $2 \mathrm{~km}$. 


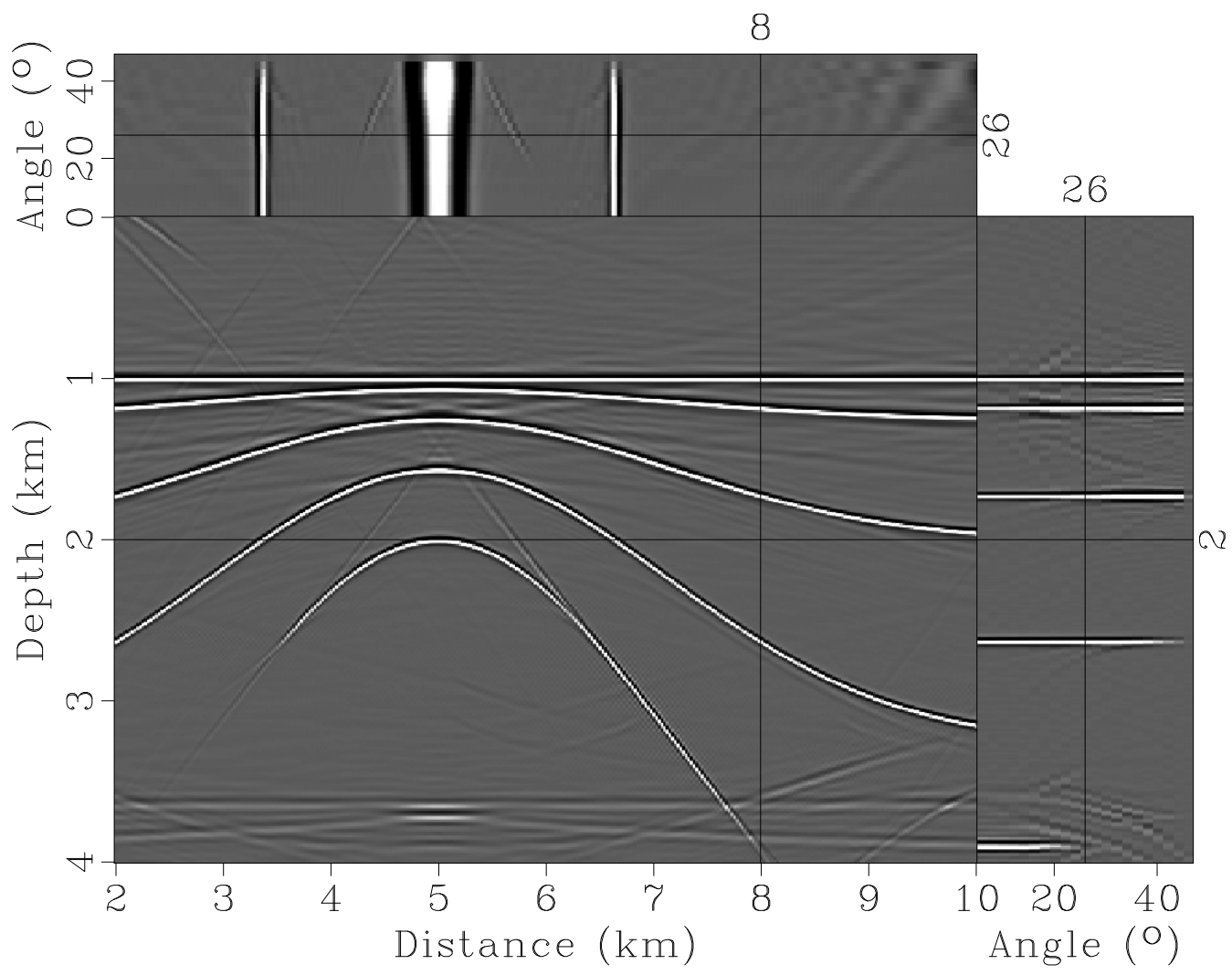

Figure 11: Migrated section after a VTI migration with velocity of $2 \mathrm{~km} / \mathrm{s}$ and $\eta=0.2$ of the VTI synthetic data in Figure 7. The angle gathers obtained, now, using the VTI mapping at $8 \mathrm{~km}$ location is displayed on the right. The top section shows a depth slice as a function of angle gather at depth $2 \mathrm{~km}$. 
reflections at their true scattering angles. In practice, the velocity model building process uses residuals along angle gathers to estimate the required velocity update. Proper definition of the angle gather residuals will simplify the update process. This is especially true if the update is based on reflection tomography.

\section{THE TTI CASE}

In the case of a tilt in the angle of symmetry of the TI (TTI) medium, the dispersion relations 2 and 3 must be altered to reflect the tilt. Specifically, the wavenumbers should be transformed to the direction of the tilt. In fact, at the reflection point all equations used to develop the mapping in equation (4) hold regardless of the direction of tilt. This implies that the quadratic solution (5) applies with $a, b$, and $c$ given by Table 1 granted that the wavenumbers are transformed in the direction of the tilt. Considering that $\phi$ is the tilt angle measured from vertical in 2-D, the horizontal (conventional) wavenumbers given by the surface-recorded data are given by

$$
k_{s c} \equiv k_{s x} \cos \phi-k_{s z} \sin \phi
$$

and

$$
k_{r c} \equiv k_{r x} \cos \phi-k_{r z} \sin \phi .
$$

where $k_{s x}$ and $k_{r x}$ now correspond to the normal-to-the-tilt wavenumber direction and they are related to $k_{s z}$ and $k_{r z}$ (tilt direction wavenumbers), respectively using equations 2 and 3. Based on the above equations, to solve for $k_{s x}$ and $k_{r x}$ needed for the angle gather mapping, we are required to solve a quartic equation that can be represented, with pain, analytically or solved numerically. Alternatively, the formulations for a transversely isotropic medium with tilt constrained to the dip (DTI), introduced by Alkhalifah and Sava (2010), is simpler than those introduced here for a general TI medium, and thus can be used at the velocity model building stage. However, when the assumption of the tilt being normal to the reflector dip fails, for example at salt flank reflections where the tilt is generally not normal to the Salt flank, we will need a general formulation similar to the one developed here.

\section{CONCLUSIONS}

We have developed analytical relationships to generate angle gathers using waveequation migration in VTI media. These relations are based on an approach for generating gathers at each depth level by converting offset-space-frequency planes into angle-space planes while simultaneously applying the imaging condition. Although the angle gathers depend on medium parameters, they are independent of the structure, which provides an opportunity for a simple and practical implementation. Comparing the mapping equation for VTI media with those for the isotropic case demonstrates the large influence that the anisotropy parameter $\eta$ has on the mapping process. On the other hand, the influence of vertical velocity is confined to the offset 
direction, and the influence of NMO velocity is small. Synthetic data applications demonstrate the importance of accurate mapping of energy in the angle domain. In the case of a TTI medium, the required modification to the mapping equations is given by the transformation of the wavenumbers in the dispersion relation to the direction of of the symmetry axis and, as a result, a similar analytic mapping exists.

\section{ACKNOWLEDGMENTS}

We are grateful to KACST, KAUST, and the Bureau of Economic Geology, University of Texas at Austin for their support. We thank Gilles Lambare, Jeff Shragge and Jun Cao for their critical and helpful review of the paper.

\begin{tabular}{|c|c|}
\hline$a$ & $\begin{array}{c}\frac{1}{2}\left(k_{h}^{2}-k_{m}^{2}\right)^{4}\left(v^{2} \eta\left(k_{h}-k_{m}\right)^{2}-2 \omega^{2}\right)^{3}\left(v^{2}(4 \eta+1)\left(k_{h}-k_{m}\right)^{2}-4 \omega^{2}\right)^{2}\left(v^{2} \eta\left(k_{h}+k_{m}\right)^{2}-2 \omega^{2}\right)^{4} \\
{\left[v_{z}^{4}\left(k_{h}^{2}-k_{m}^{2}\right)^{2}\left(-2 v^{2} \eta k_{h}^{2}\left(v^{2} \eta k_{m}^{2}+2 \omega^{2}\right)+v^{4} \eta^{2} k_{h}^{4}+\left(v^{2} \eta k_{m}^{2}-2 \omega^{2}\right)^{2}\right)\right.} \\
\left.+4 \omega^{4}\left(2 v^{2}(4 \eta+1) k_{h}^{2}\left(v^{2}(4 \eta+1) k_{m}^{2}+4 \omega^{2}\right)-v^{4}(4 \eta+1)^{2} k_{h}^{4}-\left(v^{2}(4 \eta+1) k_{m}^{2}-4 \omega^{2}\right)^{2}\right)\right]^{2}\end{array}$ \\
\hline$b$ & $\begin{array}{c}\left(k_{h}^{2}-k_{m}^{2}\right)^{4}\left(-\left(v^{2} \eta\left(k_{h}-k_{m}\right)^{2}-2 \omega^{2}\right)^{3}\right)\left(v^{2}(4 \eta+1)\left(k_{h}-k_{m}\right)^{2}-4 \omega^{2}\right)^{2}\left(v^{2} \eta\left(k_{h}+k_{m}\right)^{2}-2 \omega^{2}\right)^{4} \\
{\left[v_{z}^{4}\left(k_{h}^{2}-k_{m}^{2}\right)^{2}\left(-2 v^{2} \eta k_{h}^{2}\left(v^{2} \eta k_{m}^{2}+2 \omega^{2}\right)+v^{4} \eta^{2} k_{h}^{4}+\left(v^{2} \eta k_{m}^{2}-2 \omega^{2}\right)^{2}\right)\right.} \\
\left.+4 \omega^{4}\left(-2 v^{2}(4 \eta+1) k_{h}^{2}\left(v^{2}(4 \eta+1) k_{m}^{2}+4 \omega^{2}\right)+v^{4}(4 \eta+1)^{2} k_{h}^{4}+\left(v^{2}(4 \eta+1) k_{m}^{2}-4 \omega^{2}\right)^{2}\right)\right] \\
\left(\left(k_{h}-k_{m}\right)^{2}\left(v_{z}^{2}\left(v^{2} \eta\left(k_{h}-k_{m}\right)^{2}-2 \omega^{2}\right)+2 v^{2}(4 \eta+1) \omega^{2}\right)-8 \omega^{4}\right) \\
\left(\left(k_{h}+k_{m}\right)^{2}\left(v_{z}^{2}\left(v^{2} \eta\left(k_{h}+k_{m}\right)^{2}-2 \omega^{2}\right)+2 v^{2}(4 \eta+1) \omega^{2}\right)-8 \omega^{4}\right)\end{array}$ \\
\hline$c$ & $\begin{array}{c}\frac{1}{2}\left(k_{h}^{2}-k_{m}^{2}\right)^{4}\left(v^{2} \eta\left(k_{h}-k_{m}\right)^{2}-2 \omega^{2}\right)^{3}\left(v^{2}(4 \eta+1)\left(k_{h}-k_{m}\right)^{2}-4 \omega^{2}\right)^{2}\left(v^{2} \eta\left(k_{h}+k_{m}\right)^{2}-2 \omega^{2}\right)^{4} \\
{\left[v_{z}^{4}\left(k_{h}^{2}-k_{m}^{2}\right)^{2}\left(-2 v^{2} \eta k_{h}^{2}\left(v^{2} \eta k_{m}^{2}+2 \omega^{2}\right)+v^{4} \eta^{2} k_{h}^{4}+\left(v^{2} \eta k_{m}^{2}-2 \omega^{2}\right)^{2}\right)\right.} \\
\left.+4 \omega^{4}\left(2 v^{2}(4 \eta+1) k_{h}^{2}\left(v^{2}(4 \eta+1) k_{m}^{2}+4 \omega^{2}\right)-v^{4}(4 \eta+1)^{2} k_{h}^{4}-\left(v^{2}(4 \eta+1) k_{m}^{2}-4 \omega^{2}\right)^{2}\right)\right]^{2}\end{array}$ \\
\hline
\end{tabular}

Table 1: Exact analytical equations for the coefficients of equation (4).

\begin{tabular}{|c|c|}
\hline$a$ & $-\left(k_{h}^{2}-k_{m}^{2}\right)^{4}\left(v k_{h}-v k_{m}+2 \omega\right)^{2}\left(-v k_{h}+v k_{m}+2 \omega\right)^{2}$ \\
& $\left(-2 k_{h}^{2}\left(k_{m}^{2}\left(v^{4}-v_{z}^{4}\right)+4 v^{2} \omega^{2}\right)+k_{h}^{4}\left(v^{4}-v_{z}^{4}\right)+\left(v^{2} k_{m}^{2}-4 \omega^{2}\right)^{2}-k_{m}^{4} v_{z}^{4}\right)^{2}$ \\
\hline$b$ & $2\left(k_{h}^{2}-k_{m}^{2}\right)^{4}\left(v k_{h}-v k_{m}+2 \omega\right)^{2}\left(-v k_{h}+v k_{m}+2 \omega\right)^{2}\left(4 \omega^{2}-\left(v^{2}-v_{z}^{2}\right)\left(k_{h}-k_{m}\right)^{2}\right)$ \\
& $\left(4 \omega^{2}-\left(v^{2}-v_{z}^{2}\right)\left(k_{h}+k_{m}\right)^{2}\right)$ \\
\hline$c$ & $\left(-2 k_{h}^{2}\left(k_{m}^{2}\left(v^{4}+v_{z}^{4}\right)+4 v^{2} \omega^{2}\right)+k_{h}^{4}\left(v^{4}+v_{z}^{4}\right)+\left(v^{2} k_{m}^{2}-4 \omega^{2}\right)^{2}+k_{m}^{4} v_{z}^{4}\right)$ \\
\hline & $-\left(k_{h}^{2}-k_{m}^{2}\right)^{4}\left(v k_{h}-v k_{m}+2 \omega\right)^{2}\left(-v k_{h}+v k_{m}+2 \omega\right)^{2}$ \\
& $\left(-2 k_{h}^{2}\left(k_{m}^{2}\left(v^{4}-v_{z}^{4}\right)+4 v^{2} \omega^{2}\right)+k_{h}^{4}\left(v^{4}-v_{z}^{4}\right)+\left(v^{2} k_{m}^{2}-4 \omega^{2}\right)^{2}-k_{m}^{4} v_{z}^{4}\right)^{2}$ \\
\hline
\end{tabular}

Table 2: Exact analytical equations for the coefficients of equation (4) in the case of elliptic anisotropy $(\eta=0)$. 


\begin{tabular}{|c|r|}
\hline$a$ & $-\left(k_{h}^{2}-k_{m}^{2}\right)^{4}\left(v k_{h}-v k_{m}+2 \omega\right)^{2}\left(-v k_{h}+v k_{m}+2 \omega\right)^{2}\left(v^{2}\left(k_{h}^{2}+k_{m}^{2}\right)-2 \omega^{2}\right)^{2}$ \\
\hline$b$ & $\left(k_{h}^{2}-k_{m}^{2}\right)^{4}\left(v k_{h}-v k_{m}+2 \omega\right)^{2}\left(-v k_{h}+v k_{m}+2 \omega\right)^{2}$ \\
& $\left(v^{2}\left(-2 k_{h}^{2}\left(v^{2} k_{m}^{2}+2 \omega^{2}\right)+v^{2} k_{h}^{4}+v^{2} k_{m}^{4}-4 \omega^{2} k_{m}^{2}\right)+8 \omega^{4}\right)$ \\
\hline$c$ & $-\left(k_{h}^{2}-k_{m}^{2}\right)^{4}\left(v k_{h}-v k_{m}+2 \omega\right)^{2}\left(-v k_{h}+v k_{m}+2 \omega\right)^{2}\left(v^{2}\left(k_{h}^{2}+k_{m}^{2}\right)-2 \omega^{2}\right)^{2}$ \\
\hline
\end{tabular}

Table 3: Exact analytical equations for the coefficients of equation (4) in the case of isotropy $\left(\eta=0, v_{z}=v\right)$.

\section{REFERENCES}

Alkhalifah, T., 1995, Efficient synthetic-seismogram generation in transversely isotropic, inhomogeneous media: Geophysics, 60, 1139-1150.

— 1998, Acoustic approximations for processing in transversely isotropic media: Geophysics, 63, 623-631.

— , 2000, Prestack phase-shift migration of separate offsets: Geophysics, 65, 11791194.

Alkhalifah, T., S. Fomel, and B. Biondi, 2001, The space-time domain:theory and modelling for anisotropic media: Geophysical Journal International, 144, 105-113.

Alkhalifah, T., and P. Sava, 2010, A transversely isotropic medium with a tilted symmetry axis normal to the reflector: Geophysics, 75, A18-A24.

Alkhalifah, T., and I. Tsvankin, 1995, Velocity analysis for transversely isotropic media: Geophysics, 60, 1550-1566.

Biondi, B., 2007, Angle-domain common-image gathers from anisotropic migration: Geophysics, 72, 581-591.

Biondi, B., and W. Symes, 2004, Angle-domain common-image gathers for migration velocity analysis by wavefield-continuation methods: Geophysics, 69, 1283-1298.

de Bruin, C. G. M., C. P. A. Wapenaar, and A. J. Berkhout, 1990, Angle-dependent reflectivity by means of prestack migration: Geophysics, 55, 1223-1234.

Fomel, S., 2004, Theory of 3D angle gathers in wave-equation imaging: 74th Ann. Internat. Mtg., Soc. of Expl. Geophys., 1053-1056.

Mosher, C., and D. Foster, 2000, Common angle imaging conditions for prestack depth migration: 70th Ann. Internat. Mtg, Soc. of Expl. Geophys., 830-833.

Rickett, J. E., and P. C. Sava, 2002, Offset and angle-domain common image-point gathers for shot-profile migration: Geophysics, 67, 883-889.

Sava, P. C., and S. Fomel, 2003, Angle-domain common-image gathers by wavefield continuation methods: Geophysics, 68, 1065-1074.

$\longrightarrow$, 2005, Coordinate-independent angle-gathers for wave equation migration, in 75th Ann. Internat. Mtg, Soc. Expl. Geophys., Expanded Abstracts: Soc. of Expl. Geophys., 2052-2055.

Soubaras, R., 2003, Angle gathers for shot-record migration by local harmonic decomposition: 73rd Ann. Internat. Mtg., Soc. of Expl. Geophys., 889-892.

Stolk, C., and M. V. de Hoop, 2006, Seismic inverse scattering in the downward continuation approach: Wave Motion, 43, 579-598. 
Xie, X. B., and R. S. Wu, 2002, Extracting angle domain information from migrated wavefield, in 72nd Ann. Internat. Mtg, Soc. Expl. Geophys., Expanded Abstracts: Soc. of Expl. Geophys., 1360-1363. 